Article (refereed)

Blake, J. R.; Packman, J. C.. 2008 I dentification and correction of water velocity measurement errors associated with ultrasonic Doppler flow monitoring. Water and Environment Journal, 22 (3). 155-167. doi: 10.1111/j.1747-6593.2007.00089.x

Journal compilation @ 2008 CIWEM

This version available at http://nora.nerc.ac.uk/3203/

NERC has developed NORA to enable users to access research outputs wholly or partially funded by NERC. Copyright and other rights for material on this site are retained by the authors and/or other rights owners. Users should read the terms and conditions of use of this material at http://nora.nerc.ac.uk/policies.html\#access

This document is the author's final manuscript version of the journal article, incorporating any revisions agreed during the peer review process. Some differences between this and the publisher's version remain. You are advised to consult the publisher's version if you wish to cite from this article.

http://www3.interscience.wiley.com/journal/118500923/home 


\title{
Identification and correction of water velocity measurement errors associated with ultrasonic Doppler flow monitoring
}

\author{
J. R. Blake* and J. C. Packman
}

\section{Centre for Ecology \& Hydrology, Wallingford, Oxfordshire, UK}
Accepted for publication in Water and Environment Journal (doi: 10.1111/j.1747-6593.2007.00089.x)

Chartered Institution of Water and Environmental Management Blackwell Publishing

\section{* Corresponding Author}

J. R. Blake, Centre for Ecology \& Hydrology, Maclean Building, Crowmarsh Gifford, Wallingford, Oxfordshire, OX10 8BB, UK

Email: jarib@ceh.ac.uk

Phone: +44 (0)1491 692432 


\begin{abstract}
Ultrasonic Doppler flow monitoring (UDFM) is used to measure water flow in pipes and channels. However, a lack of scattering particles and signal noise can cause velocity errors, particularly for smaller discharges and surface water ('clean') flows. A post-processing methodology is presented that identifies and corrects these errors, maximising the value of existing data. Test criteria are used to identify errors. The error correction procedure defines depth-velocity relationships from cleaned 'training data' representing the range of flow conditions (including backed up) and uses these relationships to automatically replace erroneous velocities. UDFM velocity errors have been successfully identified and corrected in example applications. Routine use allows early identification of changes in instrument or site behaviour. The methodology is practical, consistent and updateable. This is a significant advancement on previous methods for correcting velocity errors, improving the applicability of UDFM.
\end{abstract}

\title{
Key words
}

Correction, Error, Flow, Hydrometry, Monitoring, River, Sewer, Velocity 


\section{Introduction}

Ultrasonic Doppler flow monitoring (UDFM) is a standard method for measuring water flow in pipes and channels (WRc, 1987; BSI, 1992; Herschy, 1995; Unidata, 2000; ADS, 2007). The instrumentation typically comprises of an ultrasonic Doppler velocity meter and a depth measuring pressure transducer, both sealed in a streamlined 'mouse' that is attached to the pipe invert or channel bed. The 'mouse' is connected by cable to a control box that provides power, logging and download functions. The depth and velocity are usually logged at regular intervals, to be used in subsequent velocity-area flow calculations. This research is based upon use of the Unidata Starflow instrument to measure flows in small (1-3 ha) surface water catchments. However, the findings will be broadly applicable to other situations and alternative instruments.

BSI (1992, p. 5) describes the operation of the UDFM velocity meter: 'The acoustic signal transmitted by the meter is reflected by air in suspension and particulate matter and returned to the sensor at a different frequency (Doppler effect). The shift in frequency between the signal transmitted and that received is proportional to the liquid velocity'. Thus, successful velocity measurement requires such scattering particles in the flow (e.g. Watt and Jefferies, 1996; Unidata, 2000). Each logged velocity is an average of those velocities measured by the instrument during its sampling period (which is typically kept small to optimise battery consumption). In this study the sampling period has been increased to 7 seconds (from 2) by using larger capacity batteries and modifying the instrument firmware settings. Under very low flow conditions, for example in smaller pipes and channels or when flow is backed up by a downstream control, even with an increased sampling period there 
may still be too few scattering particles passing by the instrument to provide a reliable average velocity statistic. This is especially true of 'clean' as opposed to foul water flows. In this case the Starflow records a default error velocity (either zero or a repeat of the last reliable value, depending on the instrument settings). Unidata (2000) state that a default error velocity may also be recorded if there is: (i.) excessive noise in the sampled velocities (e.g. due to the varying field of the ultrasonic cone, multiple reflections, bias by large reflectors, stationary reflectors, bedload targets or surface ripples); (ii.) poor signal strength; or (iii.) a water level below the minimum operating depth for the velocity meter. Additionally, spurious high velocity readings can be caused by signal noise at low actual velocities although a dynamic signal filter can reduce this effect (Unidata, 2000).

This research aims to identify and correct any default error velocities or spurious high velocities present in UDFM data, especially for low volume surface water flows. A new post-processing methodology is described that maximises the value of existing UDFM depth-velocity data. Stage 1 identifies the water velocity measurement errors in a semi-automated manner. This is an evolution of WRc (1987) guidance to manually check time-series plots of raw velocity and depth for errors. Stage 2 analyses training data to define the depth-velocity relationship at each site. This is analogous to the derivation of stage-discharge rating curves (e.g. CEH, 2006; Hydro-Logic, 2006). Stage 3 automatically replaces erroneous velocities with corrected values using the depth-velocity relationship. The velocity correction may be complicated by hysteretic flow response (Packman and Hewitt, 2000), transitions between sub- and super- critical flow or shifts in the depth-velocity relationship. However, the corrected velocity should be closer to the actual velocity than the 
default error value (zero or last recorded velocity), thereby improving the velocityarea flow calculation.

Recent work by ADS (e.g. Enfinger and Kimbrough, 2004; Enfinger and Stevens, 2006) uses regression methods to fit the Manning equation to depth-velocity data. Our Stage 2 procedure is distinct from their methods as it makes no a priori assumption about the nature of the depth-velocity relationship. Indeed, it would not be possible to fit a Manning equation to some of the data presented below. Furthermore, the ADS output scattergraphs with Manning curves are used to establish sewer capacity and infer flow behaviour, whereas we use the derived depth-velocity relationships to correct erroneous velocities.

Current WRc (1987) guidance indicates that flow depths under $100 \mathrm{~mm}$, or velocities under $200 \mathrm{~mm} / \mathrm{s}$, are too small for accurate measurement. Watt and Jefferies (1996) also note the inaccuracy of some UDFM devices at low velocities. However, sometimes such low flows are of interest, for example inter-event flows within sustainable drainage systems or stream baseflow. Furthermore, sites may need to be monitored despite not having optimal flow characteristics. The procedures presented here will therefore improve the applicability of UDFM.

\section{Data processing methodology}

\section{Monitoring site descriptions}

The following sections make reference to two example monitoring sites: (i.) Site A is an ordinary watercourse box culvert ( $2.44 \mathrm{~m}$ wide by $0.62 \mathrm{~m}$ deep). The data is logged every 2 minutes and the flow is unlikely to back up. (ii.) Site B is an oversized surface water sewer pipe (diameter $900 \mathrm{~mm}$ ) that also provides online flood storage. The data 
is logged every minute and the flow occasionally backs up and surcharges due to a downstream throttle. These sites are part of 14 UDFM locations run by CEH in the EPSRC Water Cycle Management for New Developments project (WaND, 2007).

\section{Stage 1: Identification of UDFM velocity errors}

Development of a data processing methodology for a particular site requires a sufficient length of depth-velocity data to represent the expected flow range. This is referred to herein as the training data. Assuming that the depth-velocity relationship is time invariant, more data will improve accuracy. The raw training data for Site A covers a 12 month period (see Fig. 1). The raw training data for Site B covers a 17 month period (see Fig. 2). At first sight the scatter in these plots looks excessive, but the large number of points ( 260000 Site A; 740000 Site B) masks a central tendency and systematic flow patterns. Example default error velocities have been marked on these plots. Both datasets also exhibit spurious high velocities that are delimited by the dashed lines and associated equations (velocity, $v$; depth, $d$ ). 


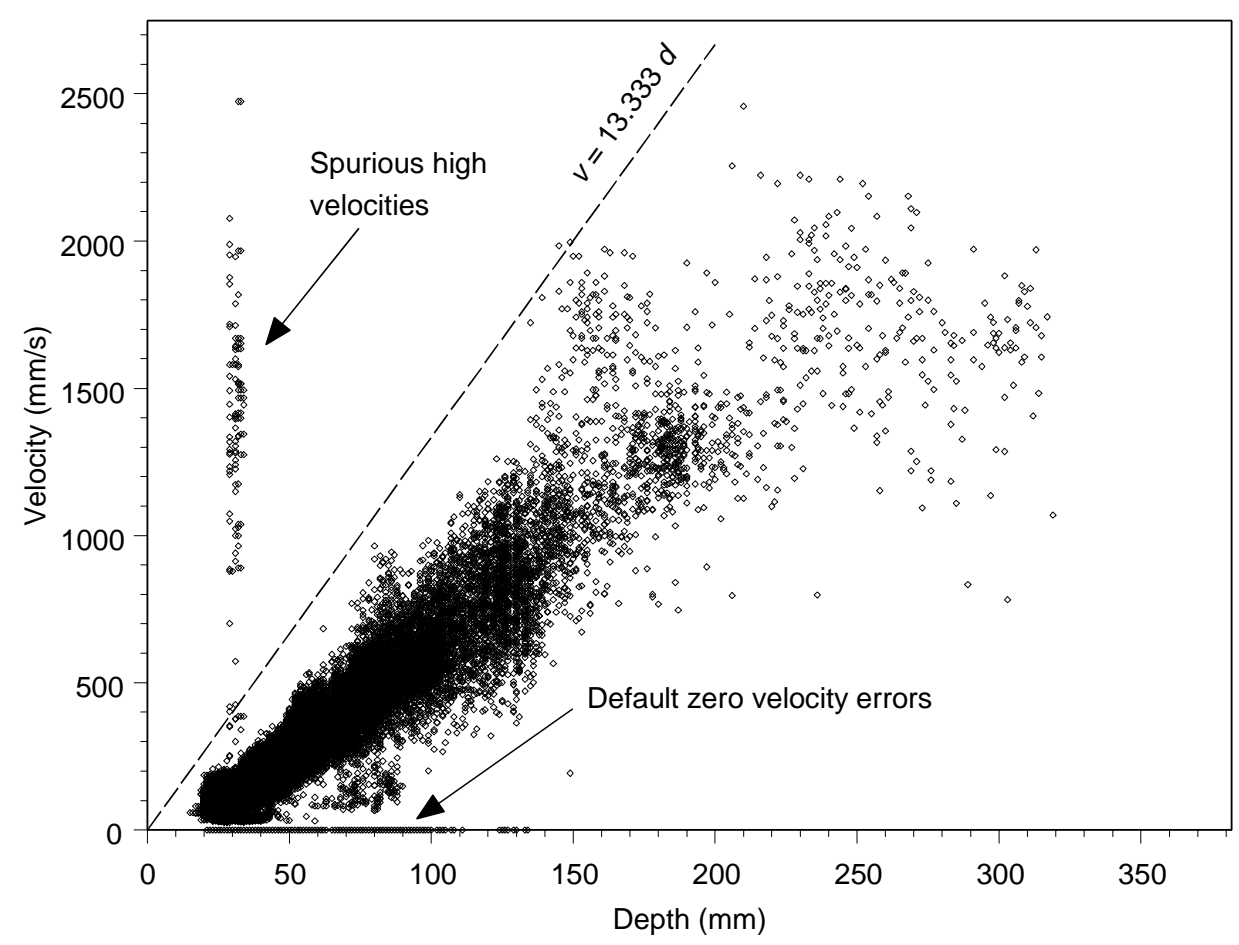

Fig. 1. Site A: Scatter plot of raw data showing typical errors.

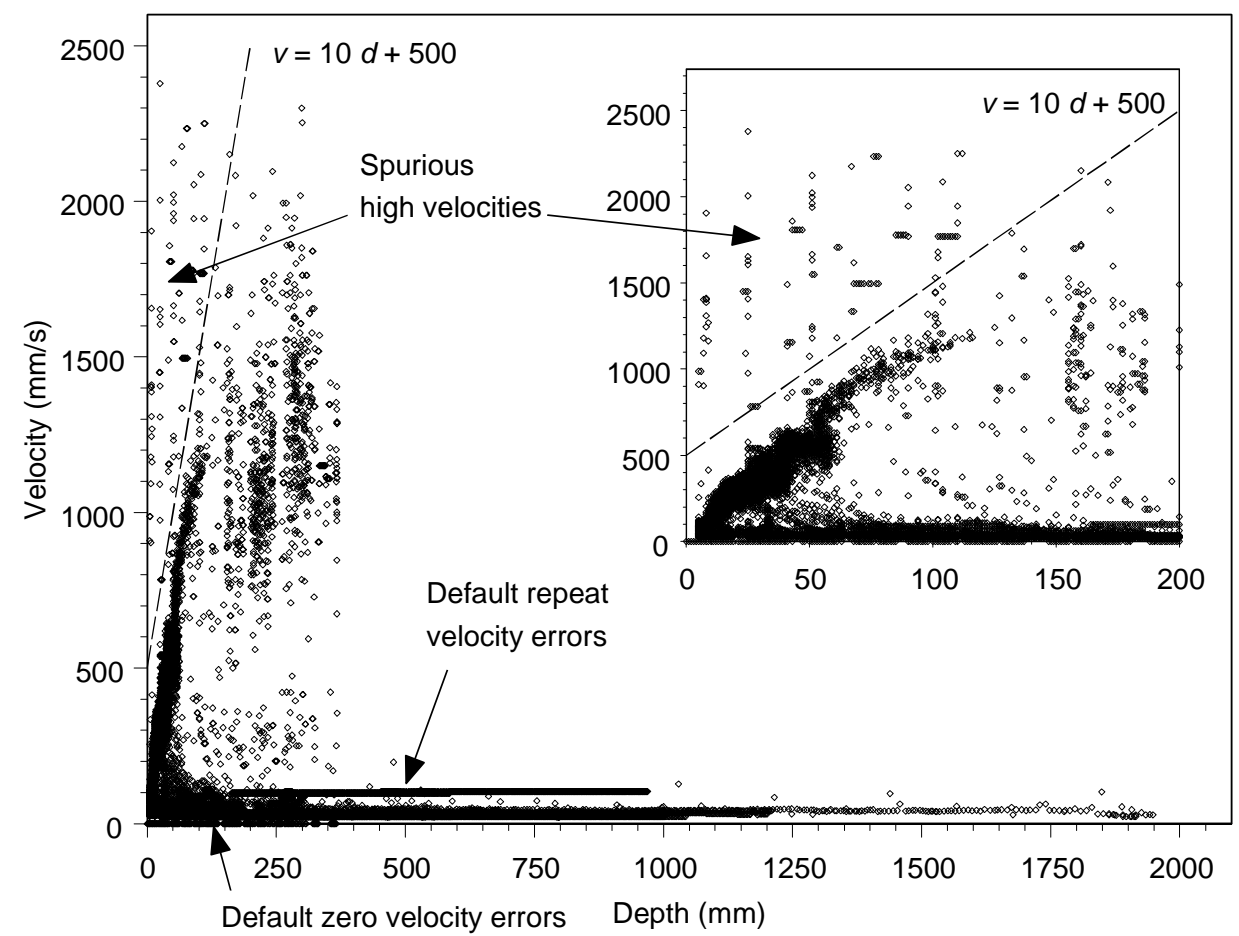

Fig. 2. Site B: Scatter plot of raw data showing typical errors, with inset detail for low depths. 
Having examined the raw training data, the following criteria can be used to identify UDFM velocity errors:

1. Default repeat velocity errors: The logged velocity (except zero) is identical to the previous logged velocity (given the $1 \mathrm{~mm} / \mathrm{s}$ velocity meter resolution and natural flow variability, a valid repeat value is unlikely).

2. Default zero velocity errors: The logged depth is greater than or equal to the minimum operating depth for the velocity meter but the logged velocity is zero.

3. Spurious high velocities: The logged velocity exceeds subjectively defined limits. For Site A: $v>13.333 d$ or $v>3000 \mathrm{~mm} / \mathrm{s}$. For Site B: $v>10 d+500$ or $v>$ $2000 \mathrm{~mm} / \mathrm{s}$. These limits balance the requirement to include valid data and the desire to exclude spurious velocities.

4. Rapid velocity increase. The logged velocity has increased by $600 \mathrm{~mm} / \mathrm{s}$ or more over one record but the depth has not doubled.

5. Lone velocity value. A seemingly valid velocity occurs in isolation from other valid readings, i.e. there is no valid reading within \pm 40 minutes of the current reading.

Criteria 1-4 have been coded in a Fortran 90 program (PROC_SF) which searches through downloaded data, identifies depth-velocity records that exhibit an error condition, replaces the erroneous velocities with an error coding value (99999) and stores the screened data in a new file. Criteria 1 and 2 are automatically evaluated. It should be noted that alternative UDFM instruments may be able to automatically flag errors due to criteria 1 and 2, e.g. ADS (2007). Criteria 3 and 4 require user-input: Potential errors are displayed along with depth-velocity values for both the preceding 
and subsequent two records. By considering the context the user can decide whether to flag the velocity as an error or not. Criterion 5 has been coded in a second program (REM_LONE_SF) that searches through the output from PROC_SF, re-codes errors and outputs the screened data to a second file. Running these programs for the example sites results in $59 \%$ of records being error coded at Site A, $31 \%$ at Site B. The large number of errors reflect the difficulties of monitoring 'clean' surface water flows in small catchments. The high error percentage for Site A is also due to a long period of low summer flows.

\section{Stage 2: Analysis of cleaned training data to define depth-velocity relationships}

It is now possible to clean the raw training data. Two programs are run sequentially: (i.) ERR_PROC_SF removes records that have an error coded velocity; and (ii.) DVZ_SF removes any remaining records that have zero velocity. These zero velocities occur when the flow is below the minimum operating depth for the velocity meter. Since the flow may not actually be zero, they must be excluded. 


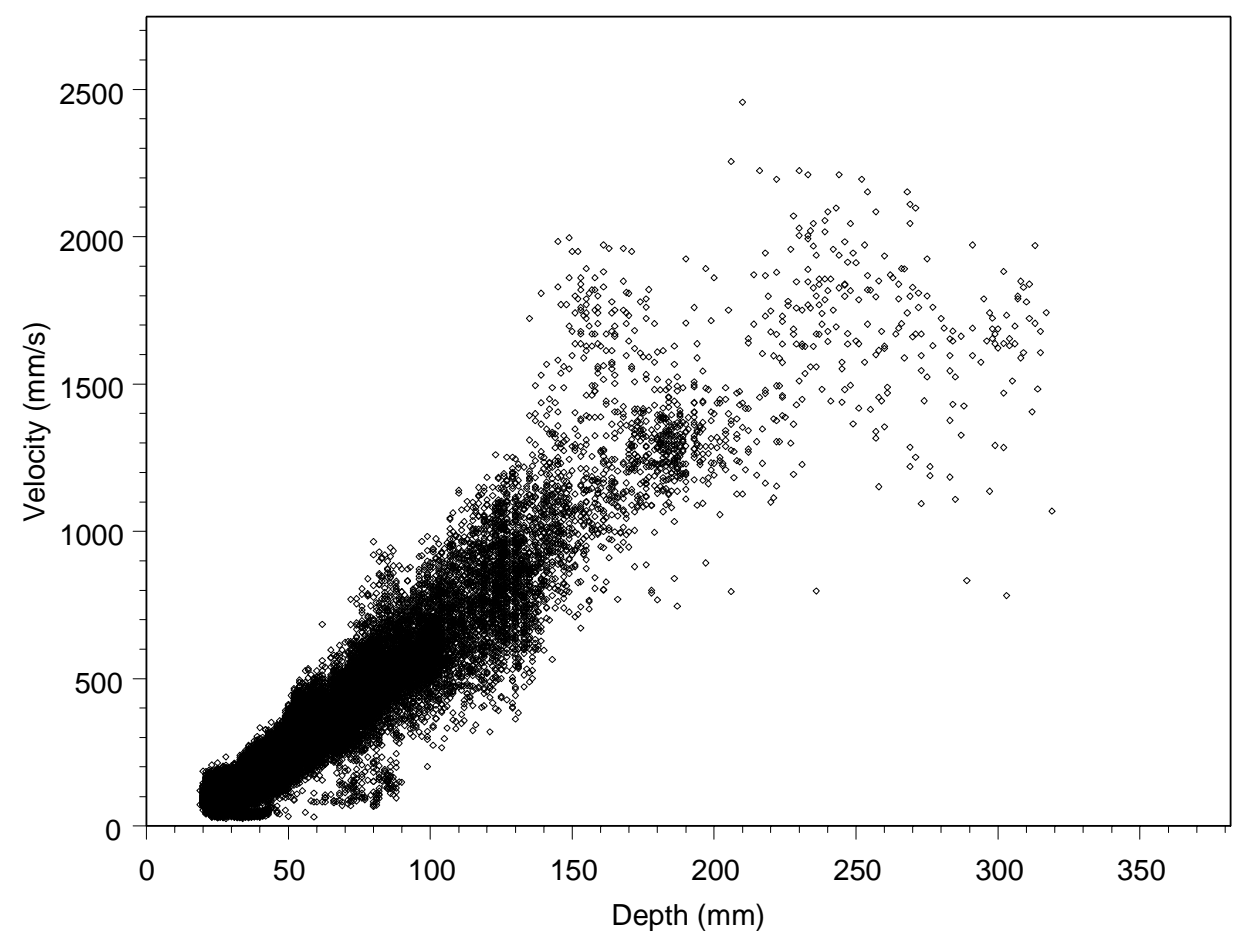

Fig. 3. Site A: Scatter plot of cleaned training data.

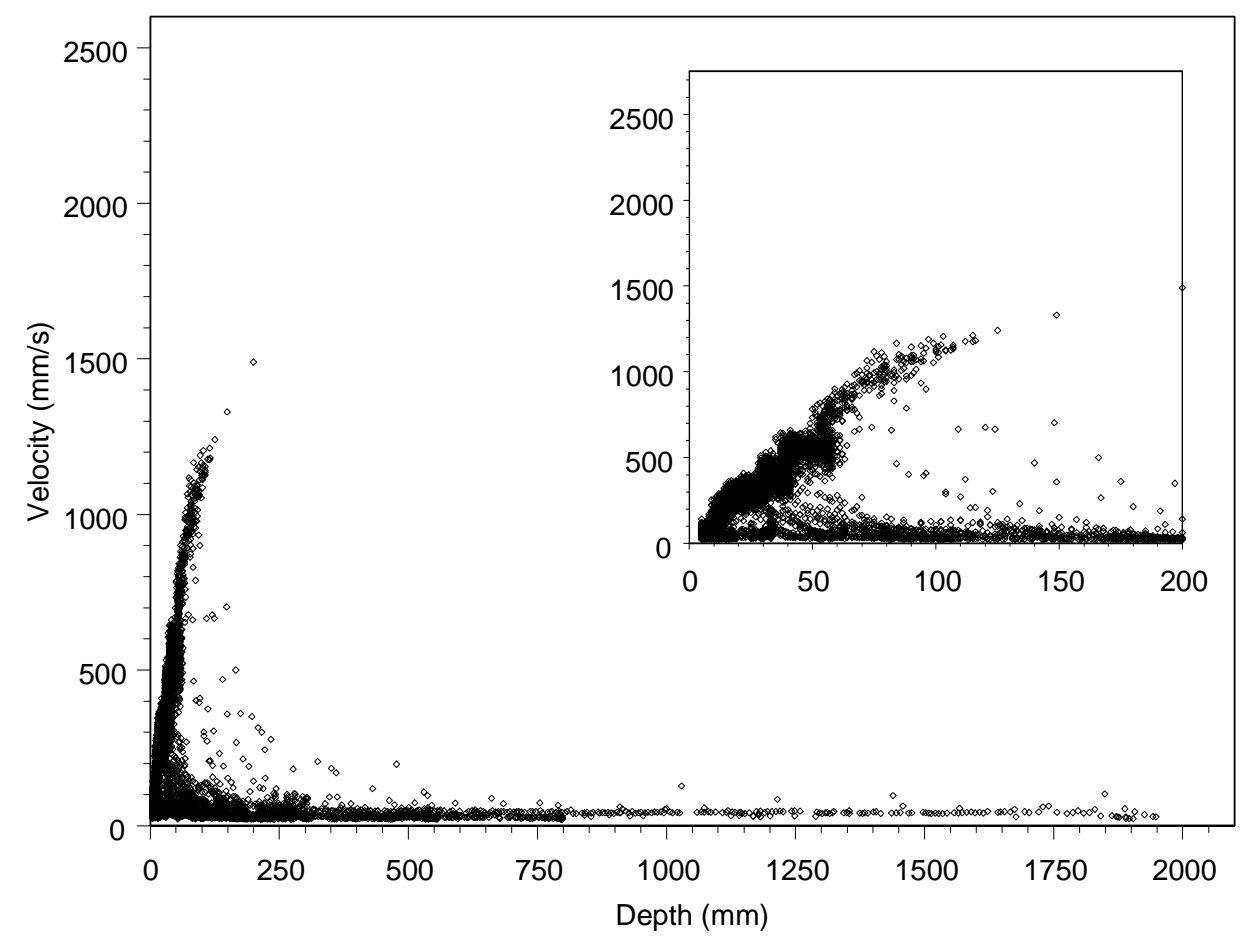

Fig. 4. Site B: Scatter plot of cleaned training data, with inset detail for low depths. 
The cleaned training data (see Figs. 3 and 4) can now be analysed to define depthvelocity relationships for use in the error correction. The Site A data ( 101000 points) exhibits a strong positive depth-velocity relationship that can be linearized by taking the logarithms of each variable (see Fig. 5). The velocity values have a relatively normal distribution, whereas the depth values are somewhat negatively skewed. Following various trials, a 'robust MM' bivariate regression, with velocity as the dependent variable, has been performed using S-PLUS 6.2 (Insightful Corp.). This was selected in preference to traditional linear regression due to the occurrence of heteroscedasticity (varying spread of residuals) and data outliers. The resultant relationship is:

$$
\operatorname{LOG}(v)=0.1727+1.3206 \operatorname{LOG}(d)
$$

The proportion of variation in response explained by this model is $90.0 \%$. Both coefficients are significant $(P<0.0000)$ and the residual standard error is \pm 0.07432 . Eq. (1) can be transformed into the power curve equation...

$$
v=1.4883 d^{1.3206}
$$

Fig. 5 shows that certain areas of the depth-velocity training data are less well represented by this equation. Lower than expected velocities may be due to meandering streamlines at lower depths, sensitivity to changing channel roughness or the velocity signals from bedload targets. Conversely, changes between sub- and super- critical flow could affect higher velocities. Refinement of the depth-velocity relationship to account for these deviations will be discussed later. 


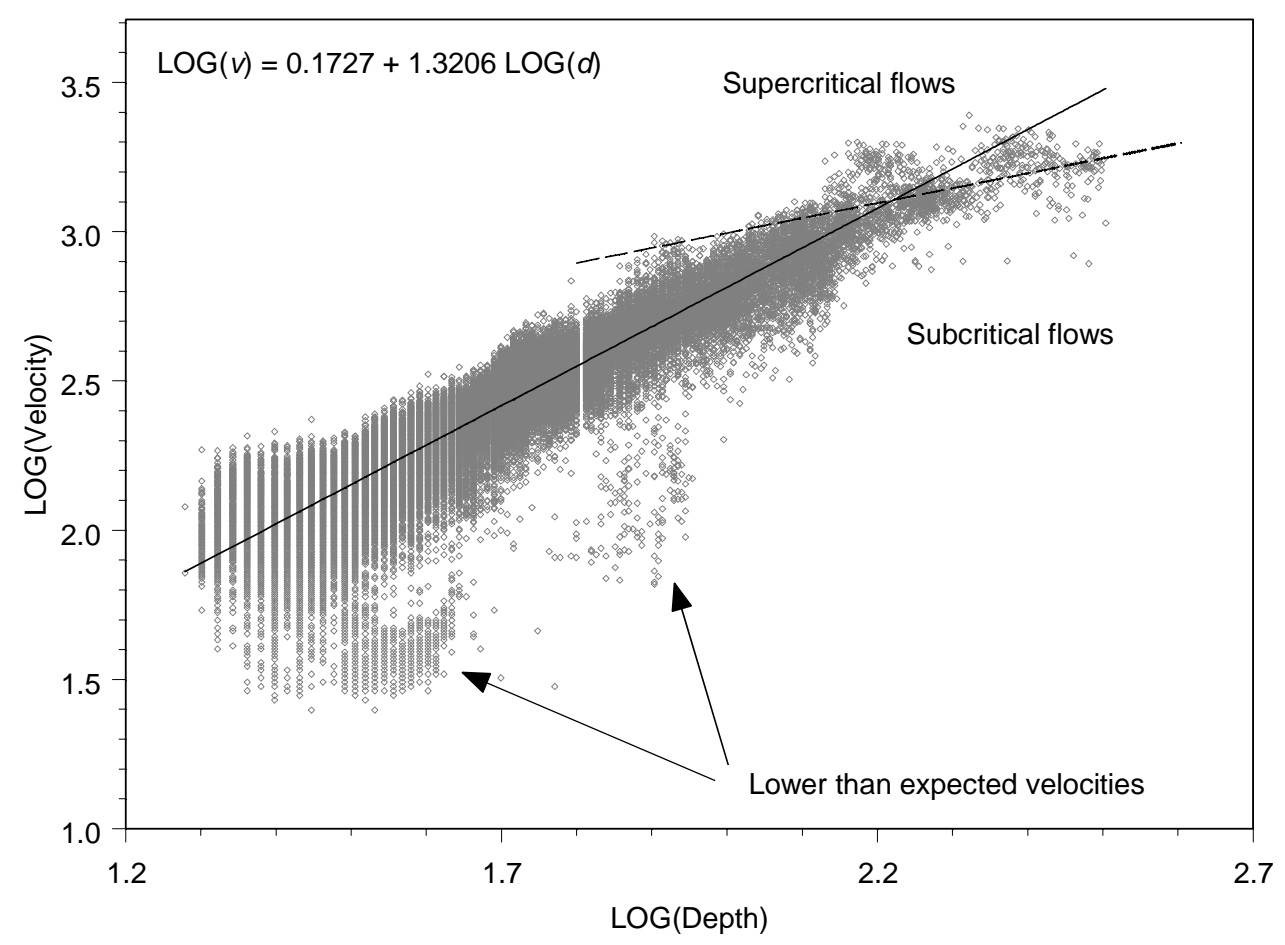

Fig. 5. Site A: Plot of cleaned training data showing the regression relationship (solid line), deviations and the sub-/super- critical flow boundary (dashed line).

To investigate scatter in the training data, the velocity time-series for a large event, covering both the rising and falling flows, was smoothed using a three point centred moving average (see Fig. 6). No persistent hysteresis loops are evident; rather the smoothed data tends towards a single relationship. Thus, the scatter is assumed to be due to noise, justifying the decision at this stage to use a single depth-velocity relationship for both rising and falling flows. 


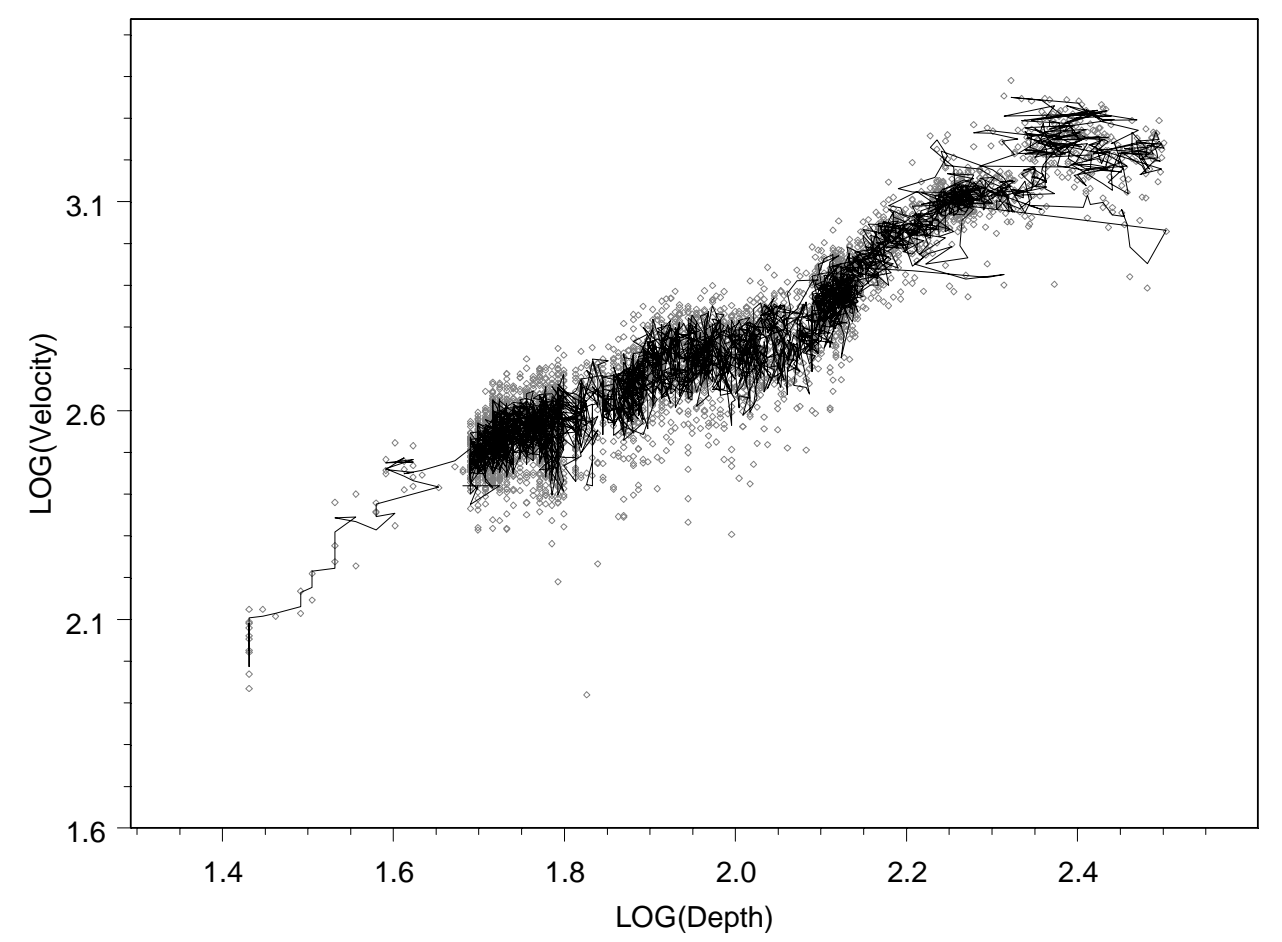

Fig. 6. Site A: Smoothed velocity time-series (line) for a large event, compared to depth-velocity data (points).

Site B exhibits a more complex depth-velocity relationship that becomes clearer on logarithmic axes (compare Figs. 4 and 7). The plot has been classified into three zones. Zone 1 represents free flow with a strong positive depth-velocity relationship. Zone 3 indicates that the system is backed up, due to the downstream throttle, and the velocity is relatively unchanging with depth. Zone 2 covers the transition between these two states. 


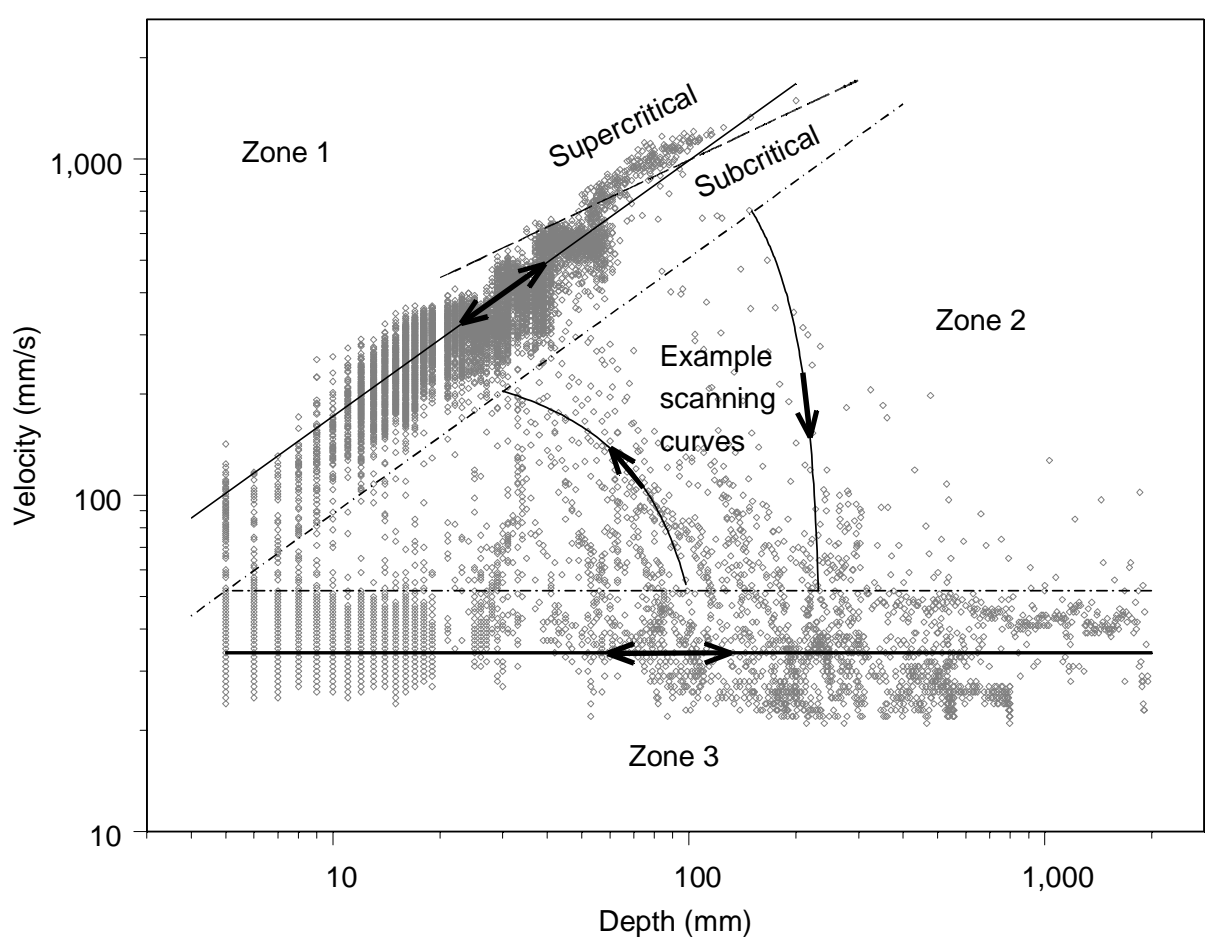

Fig. 7. Site B: Logarithmic plot of cleaned training data showing three zones (delineated by dash-dot thresholds lines), the predominant depth-velocity relationship in each zone (solid trend line(s)) and the sub-/super- critical flow boundary (dashed line).

The Zone 1 data has been extracted from the cleaned training data by examining the individual tracks between successive depth-velocity data pairs. Data points forming tracks that remained within the area perceived to be Zone 1 were retained, whilst data points forming tracks that exited Zone 1 were excluded. Although somewhat subjective, this is a useful approach given the data complexity. The Zone 1 data ( $~ 8000$ points) was linearized by taking the logarithms of each variable. Both the velocity and depth values possess a fairly normal distribution. As before, a 'robust MM' bivariate regression has been performed. The resultant Zone 1 trend equation is: 


$$
\operatorname{LOG}(v)=1.4733+0.7608 \operatorname{LOG}(d)
$$

The proportion of variation in response explained by this model is $76.2 \%$. Both coefficients are significant $(P<0.0000)$ and the residual standard error is \pm 0.07403 . Eq. (3) can be transformed into the power curve equation...

$$
v=29.737 d^{0.7608}
$$

As previously, some deviation from this relationship may be explained by the transition between sub- and super- critical flows (as marked on Fig. 7). The relationship between hydraulic radius and velocity was also investigated but this was no stronger because the Zone 1 flow depths are below half pipe, where the relationship between depth and pipe hydraulic radius is reasonably linear. The specified Zone 1 threshold line is parallel to the Zone 1 trend line and it delimits the Zone 1 values. It was calculated by adding the largest negative residual $(-0.2901)$ to the intercept term in Eq. (3), then transforming this into a power curve equation...

$$
v=15.248 d^{0.7608}
$$

The Zone 3 depth invariant relationship was determined from the low velocity histogram (Fig. 8). The backed up flow velocity has been approximated as the median value $(34 \mathrm{~mm} / \mathrm{s})$, whilst the Zone 3 threshold velocity is $52 \mathrm{~mm} / \mathrm{s}$. 


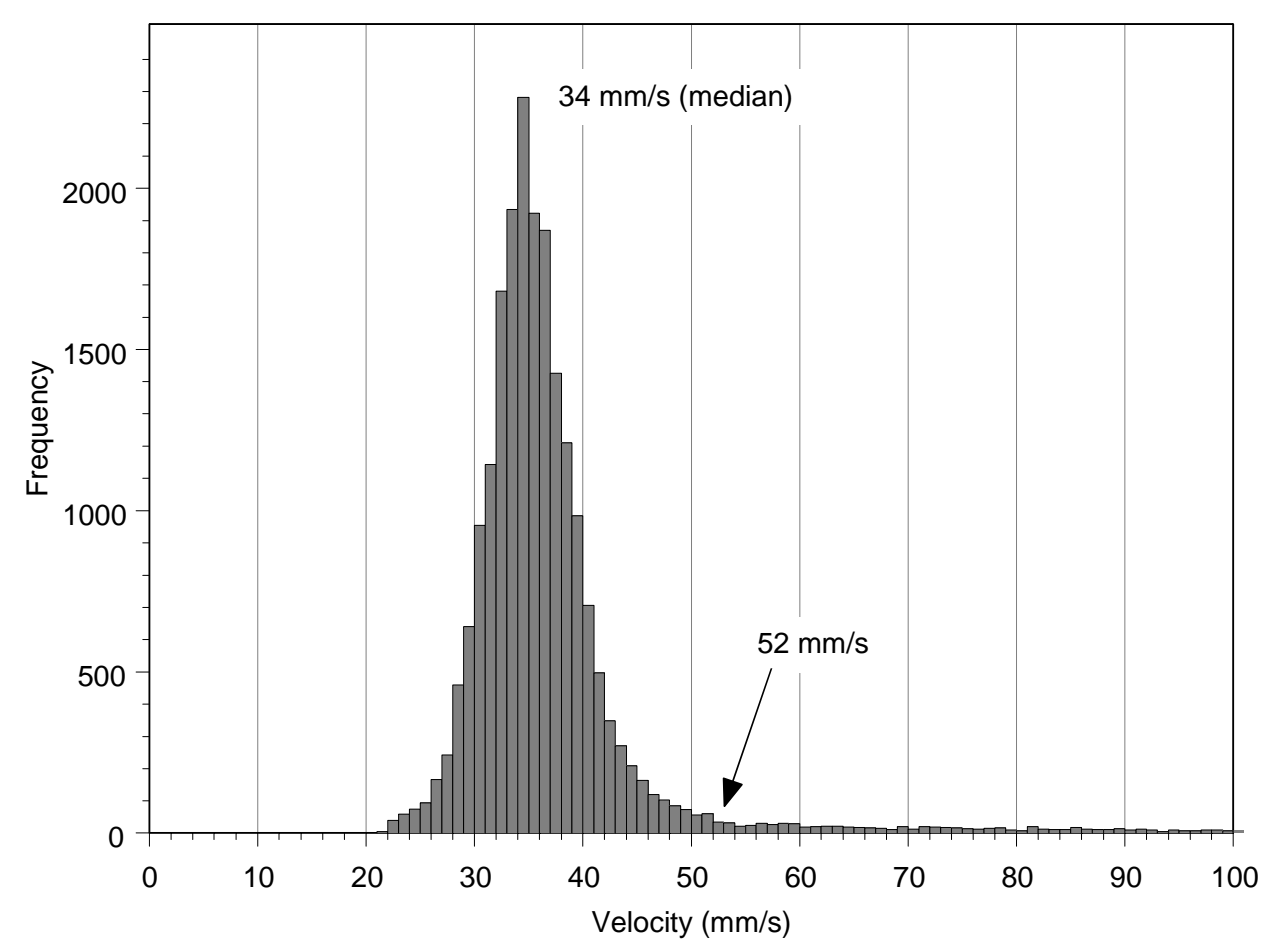

Fig. 8. Site B: Histogram of low velocities from cleaned training data.

Having established thresholds for Zones 1 and 3, the Zone 2 depth-velocity relationships were determined by examining the tracks between successive depthvelocity data points. A hysteretic negative depth-velocity relationship was revealed, which varies in magnitude with increasing or decreasing depth. This has been approximated using two average rates: (i.) Zone 2 velocity loss rate, velocity decreases by $8.0 \mathrm{~mm} / \mathrm{s}$ per unit depth increase; and (ii.) Zone 2 velocity gain rate, velocity increases by $2.2 \mathrm{~mm} / \mathrm{s}$ per unit depth decrease. These linear rates plot as curves on a logarithmic graph (hence the example scanning curves in Fig. 7).

\section{Stage 3: Correction of UDFM velocity errors}

The depth-velocity relationships defined in Stage 2 have been incorporated into INFILL_SF - a new program that automatically corrects UDFM velocity errors. This 
program processes the Stage 1 output file, replacing any error coded velocities with corrected values according to the site dependent rules. For Site A, the replacement velocity is calculated using Eq. (2). For Site B, the process is more complex (see Fig. 9) depending on the zones in which the previous record and next valid record (i.e. non-error coded velocity) occur and whether the event ends (i.e. depth below minimum operating depth) before the next valid record occurs. The Zone 1 threshold is defined by Eq. (5) and the Zone 3 threshold is $52 \mathrm{~mm} / \mathrm{s}$. The INFILL_SF program has been run on the data for both sites (see Results).

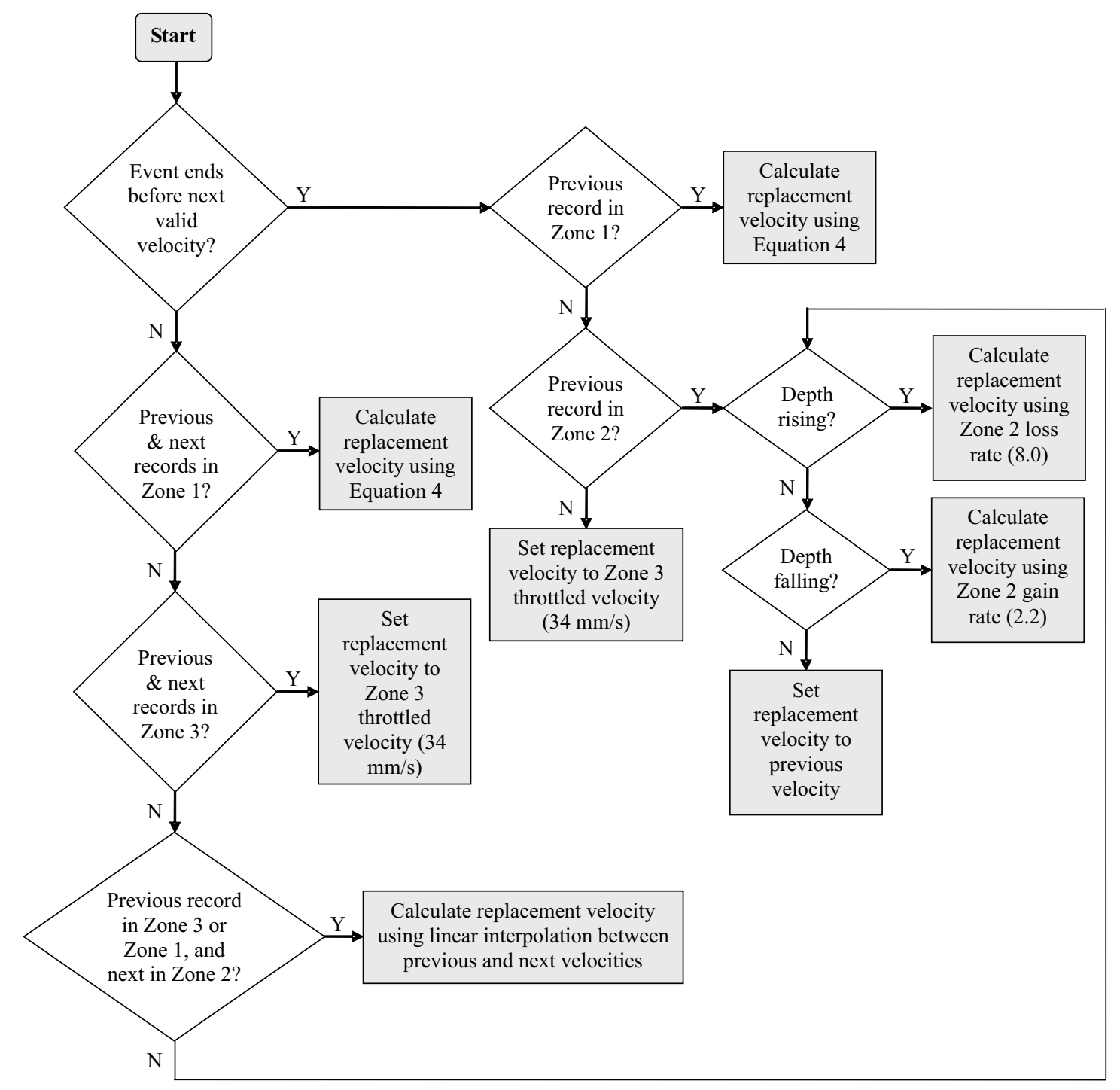

Fig. 9. Flowchart showing data correction process used in INFILL_SF for Site B. 


\section{Using the data processing programs}

A training dataset (see Stage 2) has been used to develop the data processing programs. Whilst this could encompass all data from a monitoring campaign, it can be beneficial to use the first few sets of data for training and then use the defined relationships for routine processing of each new dataset as it is acquired. Checking new data against training data can reveal instrument faults (such as depth sensor zerodrift, see Watt and Jefferies, 1996) or the need for updated depth-velocity relationships (e.g. if sediment bedload is moving through a surface water sewer system). However, to avoid errors of extrapolation, velocity correction should not extend beyond the range of the training data (instead the training data and associated relationships would need to be updated). The distinction between using the programs in training or routine mode is shown in Fig. 10. 


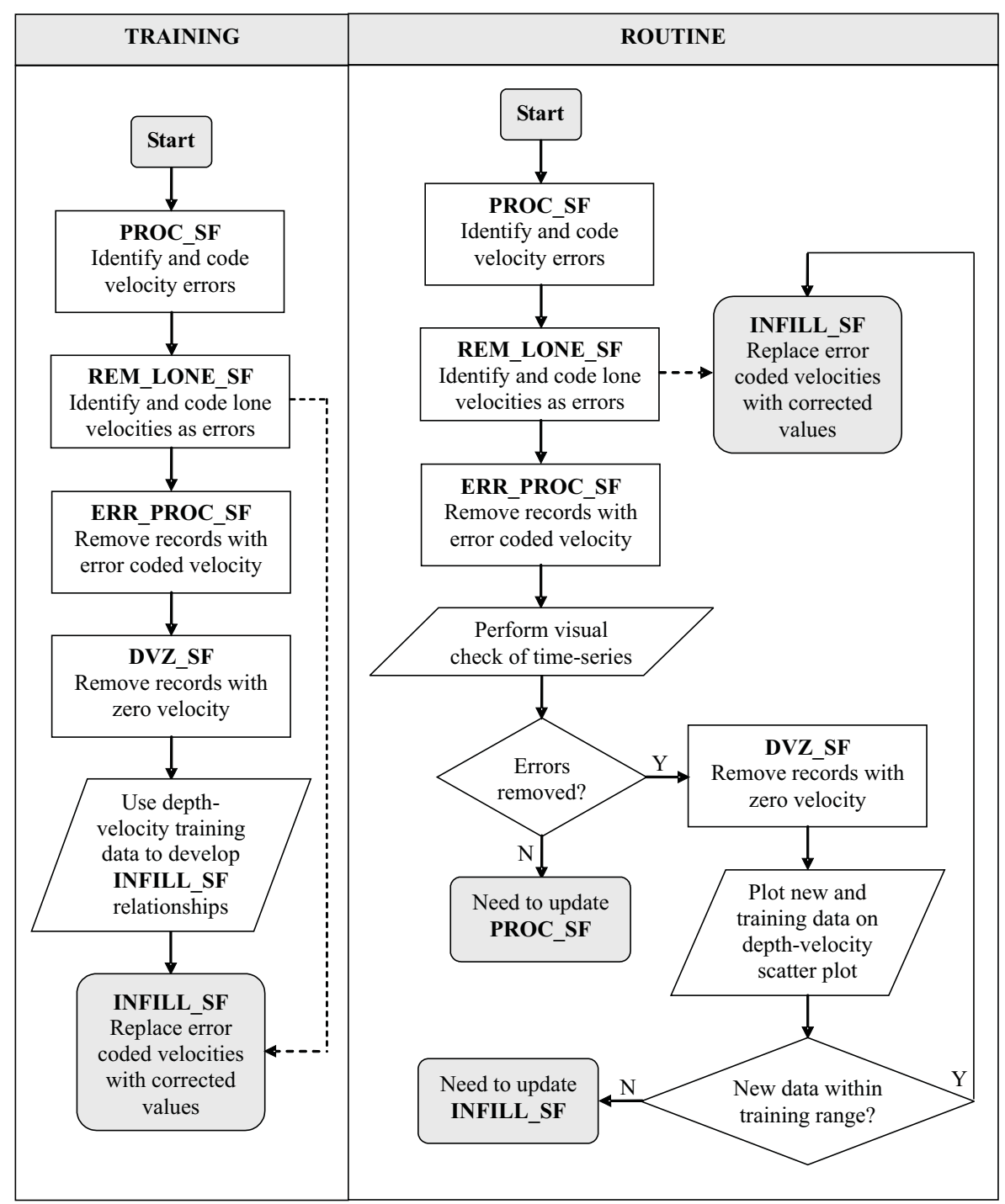

Fig. 10. Data processing program use in training and routine modes (dotted line shows use of previous output).

\section{Results and Discussion}

\section{Corrected velocity data}

The data processing methodology has been used to identify and correct UDFM water

velocity errors at example sites A and B. Selected events are shown in Figs. 11 to 13.

They represent different flow conditions and show the 'worst case' number of 
velocity errors. In all of these, and subsequent figures, raw data is plotted as filled blocks, corrected as a solid line.

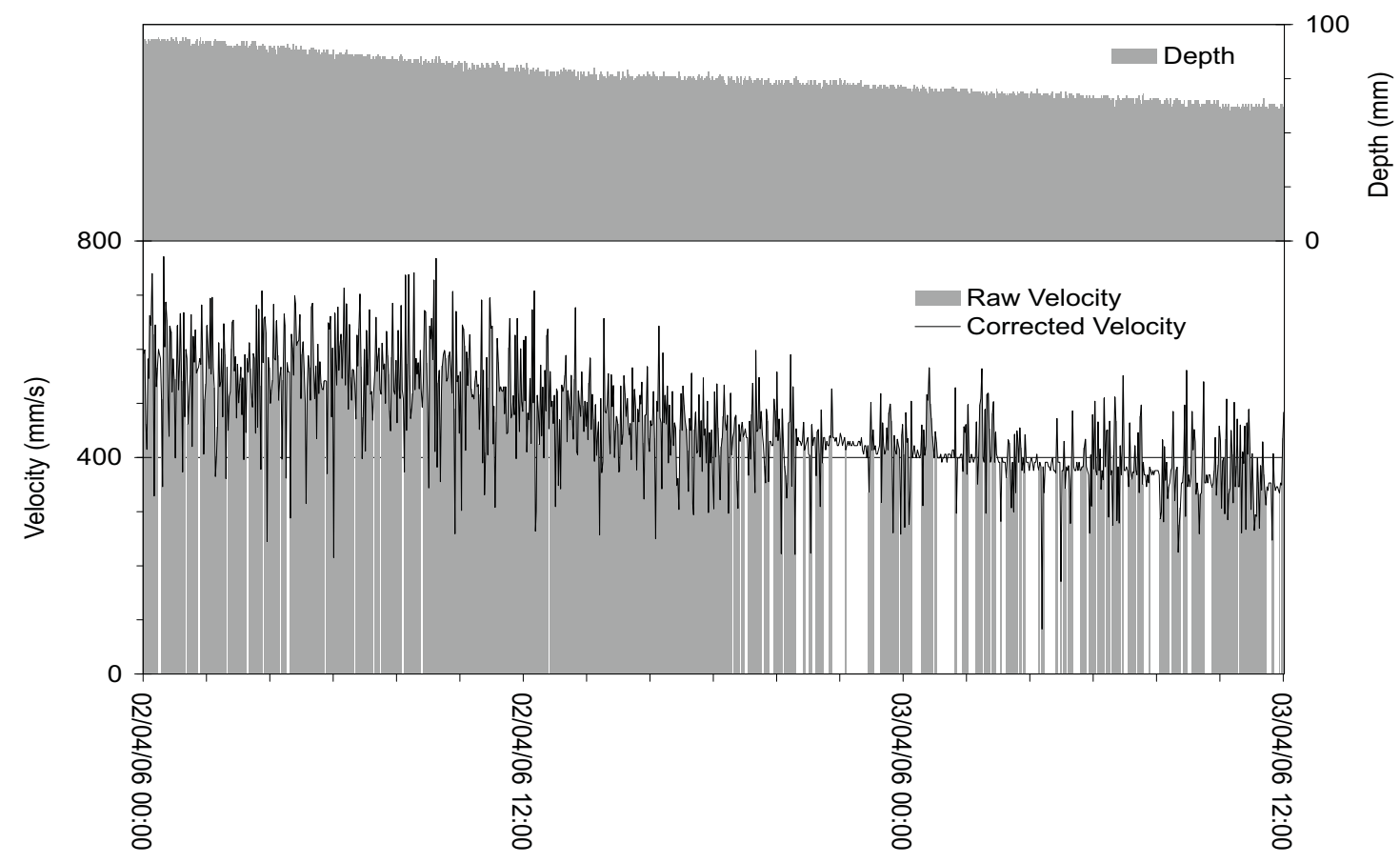

Fig. 11. Site A: Time series of depth and velocity (raw \& corrected).

Fig. 11 shows that the errors at Site A are predominantly of the 'default zero velocity' type, as indicated by the unfilled areas mainly occurring between 02/04/06 18:00 and 03/04/06 12:00. They are probably due to excessive noise in the sampled velocities. This may be linked to surface ripples affecting the Doppler return. With 30 readings per hour, 'default repeat velocity' errors are hard to spot in this plot although the program finds and corrects them. The corrected velocity is clearly an improvement over the raw data; the replacement velocities seem reasonable given their context, but are less variable than the raw data because they are derived from the less variable depth time series. 


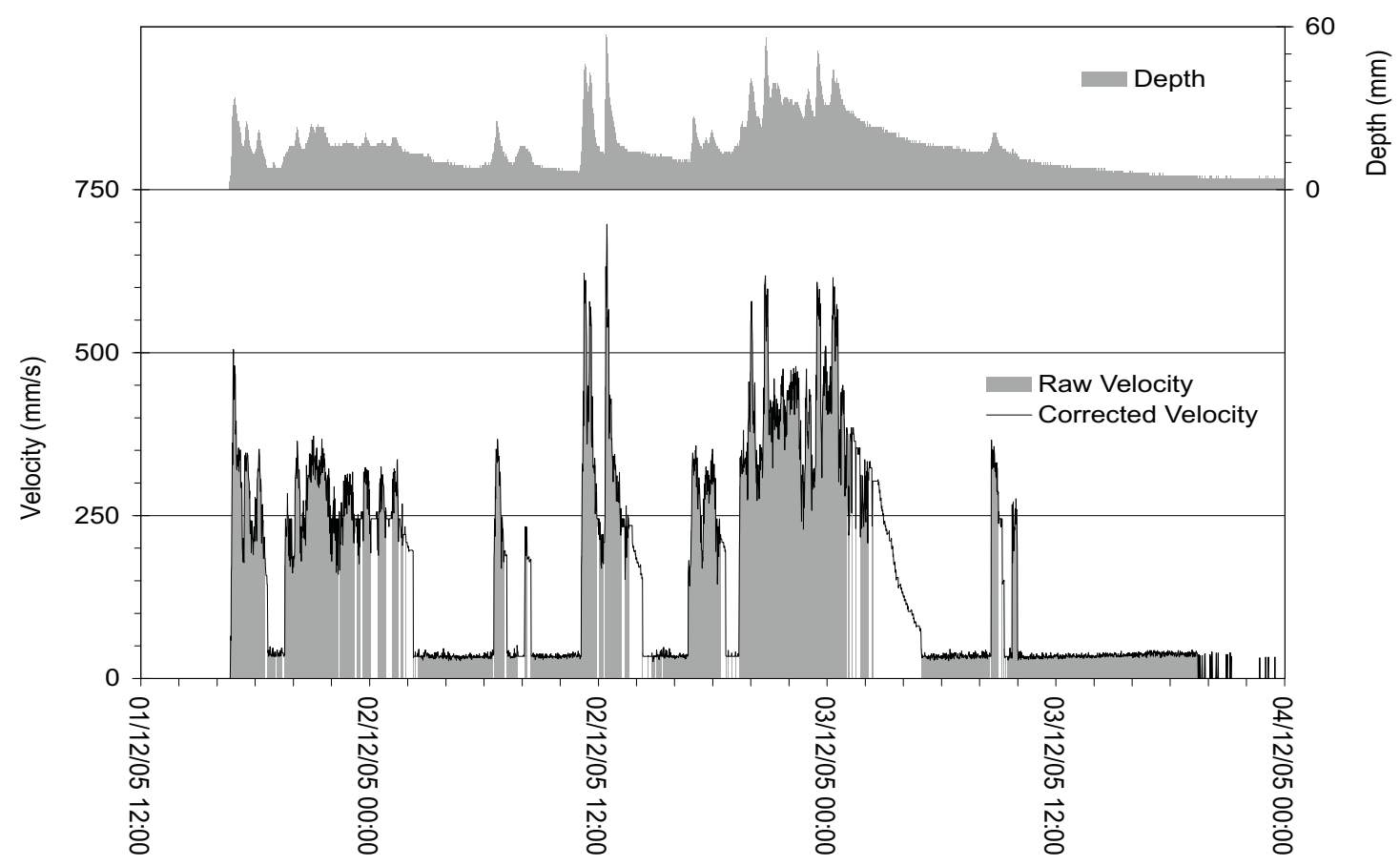

Fig. 12. Site B: Time series of depth and velocity (raw \& corrected) under normal flow conditions.

Fig. 12 shows that at Site B (the oversize pipe) the errors under normal flow conditions are predominantly of the 'default zero velocity' type, mainly occurring during flow recessions. As for Site A, this is probably due to excessive noise. Additional errors occur after 19:00 on 03/12/05 when the water level falls below the instrument minimum operating depth (these cannot be corrected). With 60 readings per hour, 'default repeat velocity' errors are again hard to spot in this plot. The corrected velocity time series shows an improvement on the raw data, with reasonable replacement values. The improvement is not as strong as for Site A due to the weaker depth-velocity relationship at Site B (see Stage 2). Thus, a step may occur between the replacement and original velocities, which is an area for future improvement. 


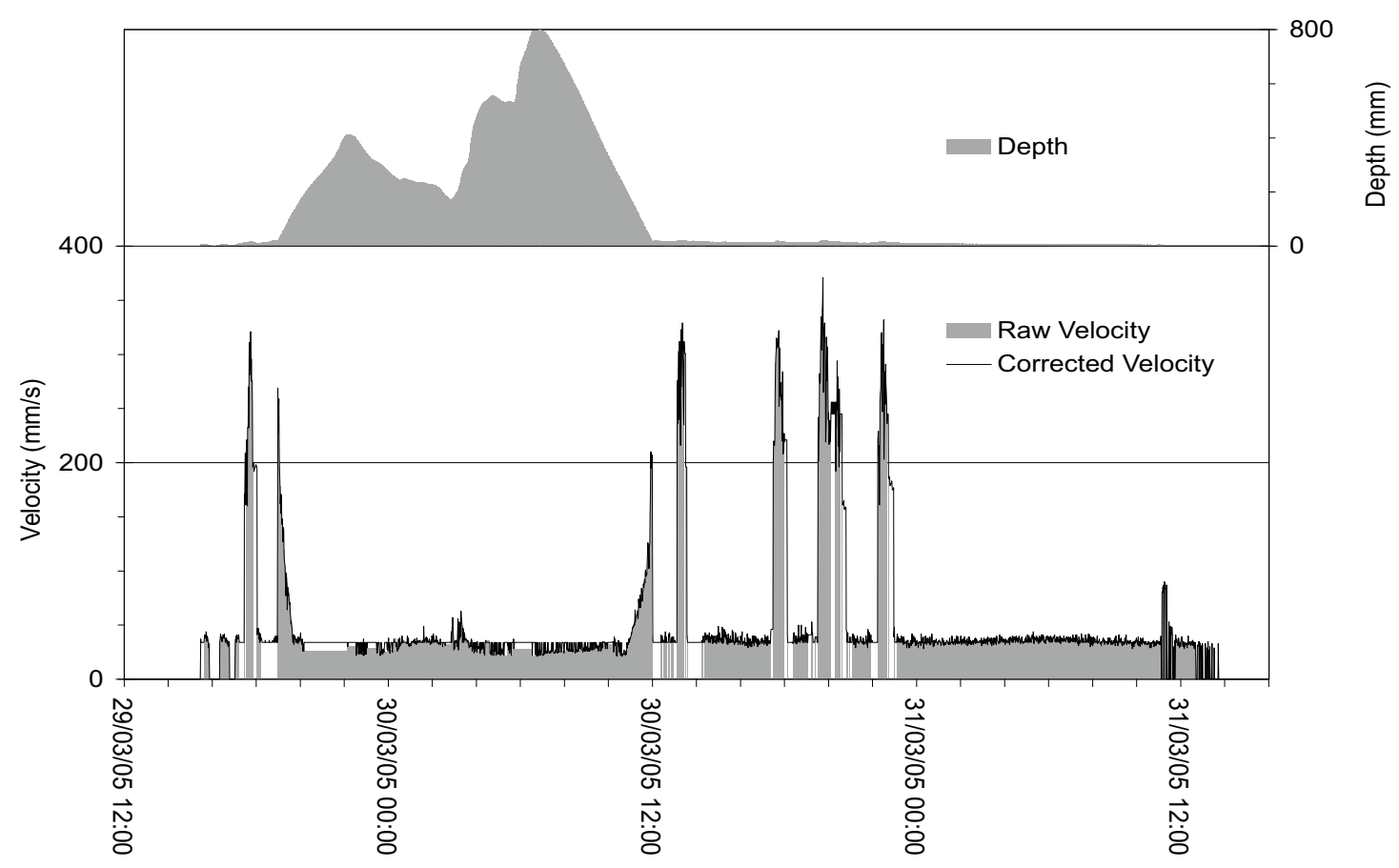

Fig. 13. Site B: Time series of depth and velocity (raw \& corrected) under backed up flow conditions.

Fig. 13 shows that at Site B the errors under backed up conditions are predominantly of the 'default repeat velocity' type (e.g. the constant raw velocities around 29/03/05 21:00). They indicate that few scattering particles are passing the sensor, presumably due to low flow velocities and deposition. Some 'default zero velocity' errors also occur and are again probably due to excessive noise. The corrected velocity time series shows a visual improvement on the raw data, but the replacement velocity for backed up flow (34 mm/s) may be somewhat high (compared to the valid raw data).

\section{Discharge calculation}

Both the raw and corrected velocity data have been used to generate discharge time series. The corrected discharge has been calculated using the corrected velocities, the raw depths and the known cross section geometry. Figs. 14 to 16 show the corrected 
discharge for the previously selected events, along with the raw discharge (calculated using raw depths and raw velocities). Although beyond the scope of this paper, such discharges should ideally be calibrated against manual flow gauging (e.g. WRc, 1987; Vermeyen, 2000) before being used in any application.

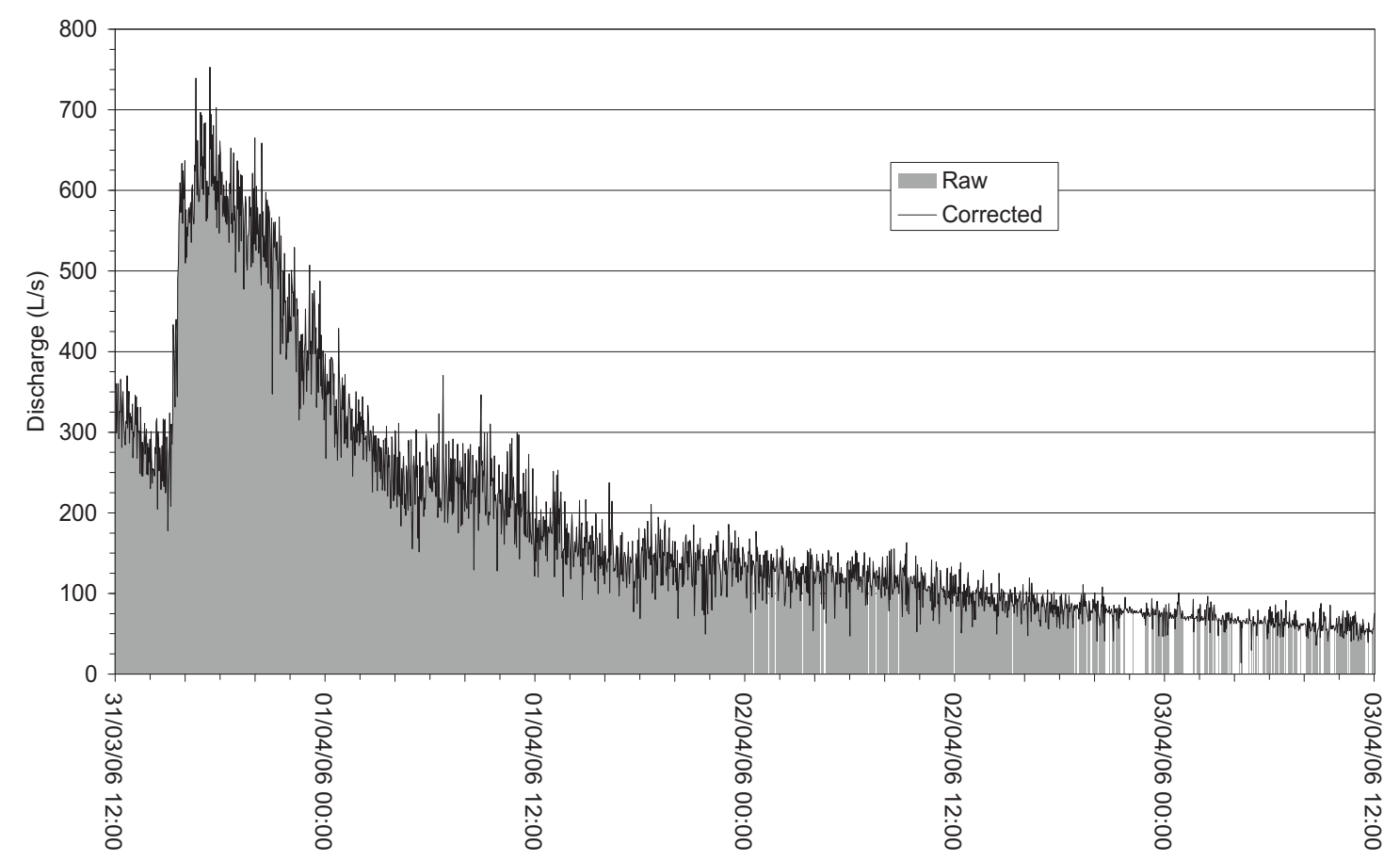

Fig. 14. Site A: Time series of discharge (raw \& corrected). 


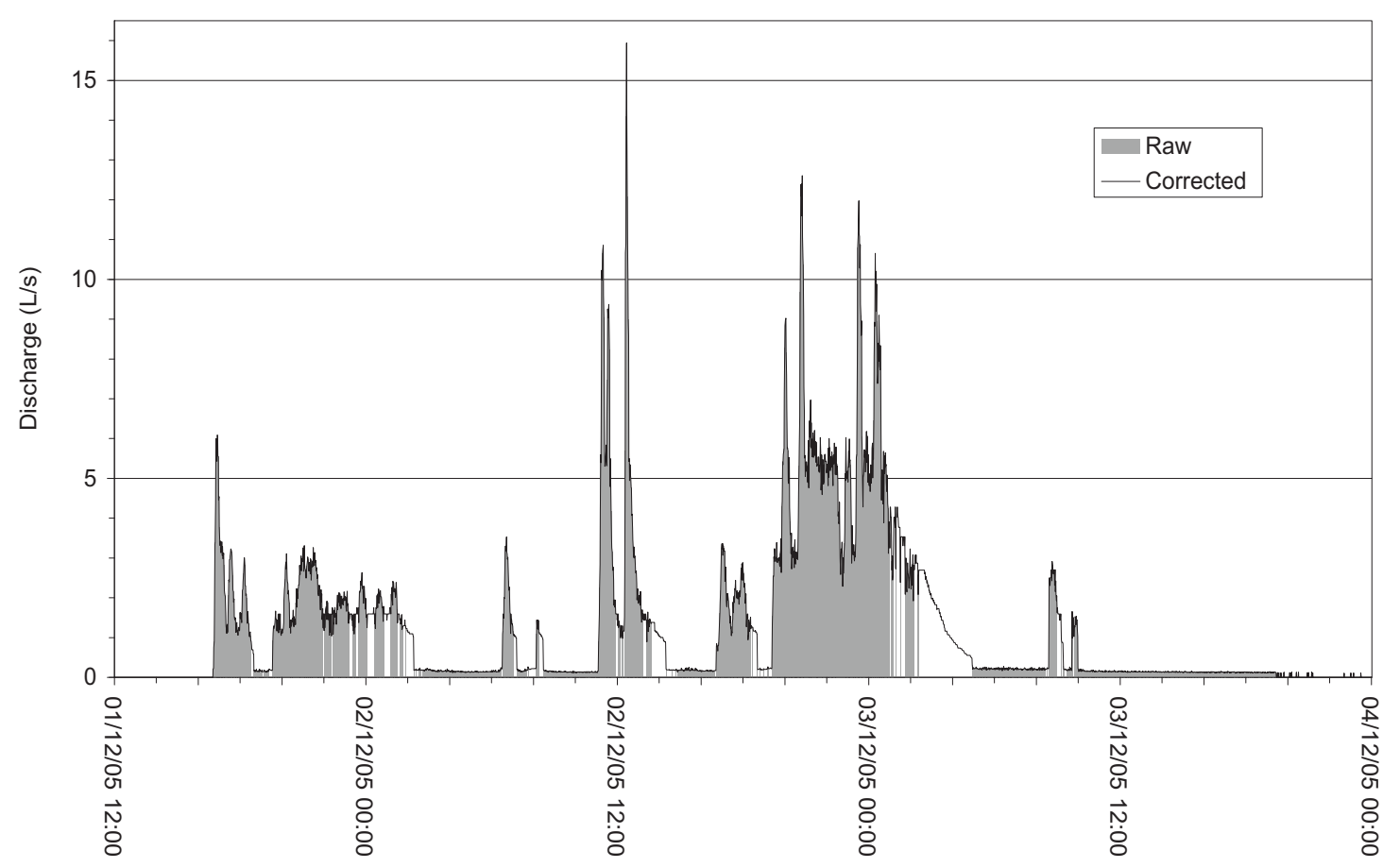

Fig. 15. Site B: Time series of discharge (raw \& corrected).

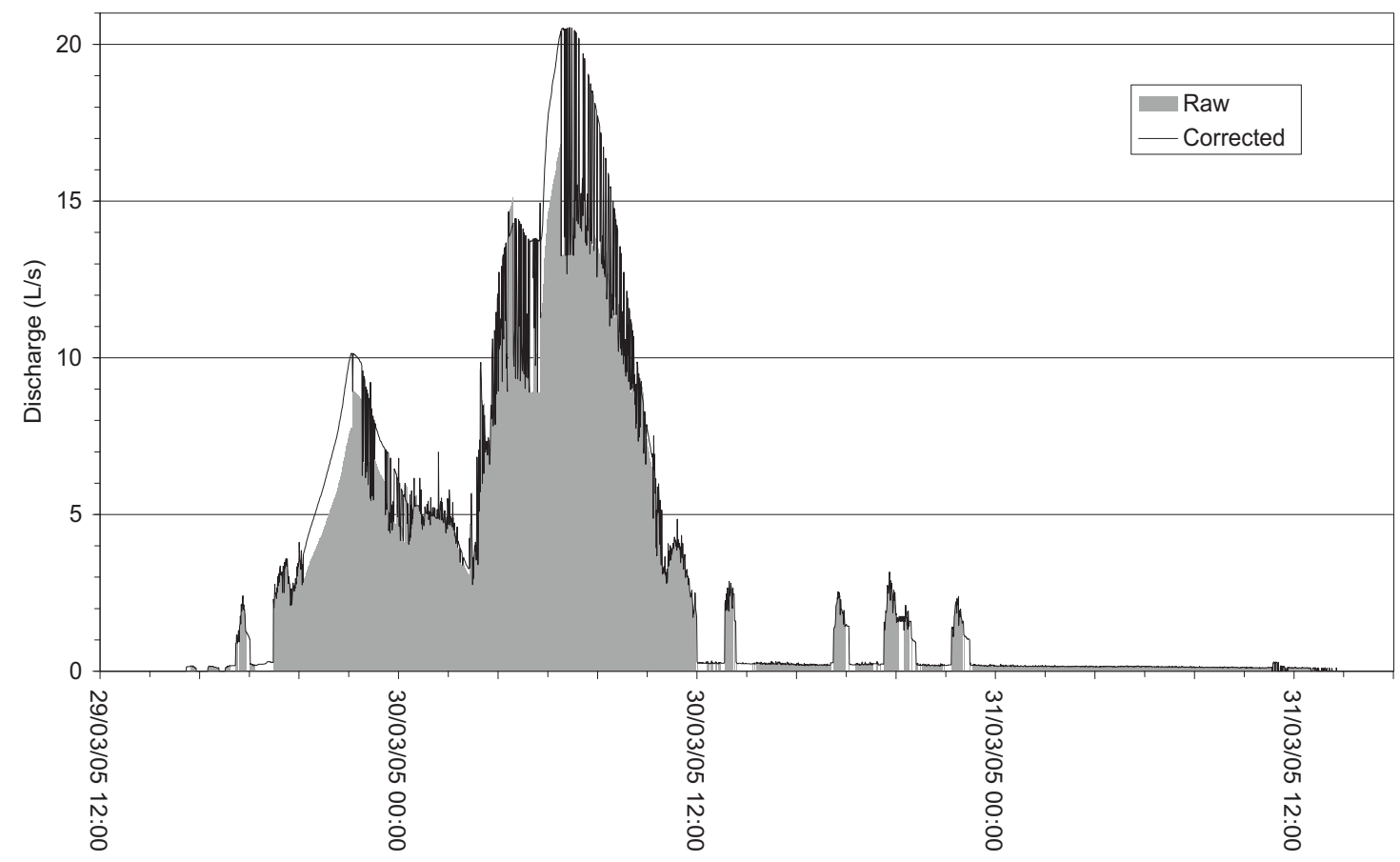

Fig. 16. Site B: Time series of discharge (raw \& corrected).

These figures highlight the benefits of the new methodology. Gaps in the discharge series, caused by 'default zero velocity' errors, have been filled and 'default repeat 
velocity' errors have been replaced, allowing improved runoff estimates. With semiautomated error identification, fully automated error correction and site dependent rules, the new methodology can correct data consistently. Furthermore, all stages of the methodology can be updated as necessary and re-run independently. This provides a significant advantage over the less consistent methods suggested in WRc (1987) guidance, namely manual checking of raw velocity and depth time-series plots for erroneous data, and using manual lines drawn on flow-depth scattergraphs to obtain replacement flow values. It should be noted that the estimates of corrected discharge under backed up conditions (Fig. 16) may be somewhat high at times, due to the use of a fixed replacement velocity.

\section{Evaluation of methodology}

In the preceding sections, the new methodology has been evaluated qualitatively. For example, replacement velocities are noted as 'reasonable given their context' (the context being the preceding and subsequent values in the data). This approach has been necessary due to the inherent difficulties of quantitative evaluation. For example, whilst the variation in response statistic aids in the assessment of alternative depthvelocity relationships, it is less useful for evaluating the methodology itself. Furthermore, although confidence limits could be calculated for erroneous velocities replaced using a regression calculation, they would not be possible for non-regression areas of the depth-velocity relationship (e.g. Zones 2 and 3 in Fig. 7). A quantitative evaluation could involve replacing a proportion of a known error free dataset with realistically distributed erroneous velocities, then performing error identification and correction to produce a corrected dataset. This corrected dataset could be compared with the original to quantify the match between corrected and actual velocities. 
However, since velocity errors tend to occur during flow recession or backed up conditions, it has not been possible to obtain an error free dataset for these conditions. Thus we have had to rely on a qualitative evaluation of the methodology.

\section{Future directions}

Future work could improve the depth-velocity relationships used in the velocity correction procedures. This might parallel recent developments in the derivation of stage-discharge ratings (e.g. Petersen-Overleir, 2006). Currently, the relationships (and their accuracy) are biased towards the more numerous lower depth-velocity readings. An upgrade to multi-part regressions could improve representation of the sub- to super- critical flow transition (e.g. see Fig. 5), enabling the more accurate correction of the albeit infrequent errors at higher velocities. Following Enfinger and Schutzbach (2005), it might also be possible to explicitly account for the effects of varying channel roughness. Error correction for backed up conditions (e.g. Zone 3 in Fig. 7) might be improved with a variable depth-velocity relationship (rather than a fixed value). Since error correction is predominantly required on falling flows, it might be improved by defining individual depth-velocity relationships for rising or falling flows. Depth-velocity relationships that are non-stationary in time, e.g. for natural channels or when a sediment pulse is moving slowly through a drainage system, will require time variant correction.

\section{Conclusions}

(1) A new post-processing methodology has been presented that identifies and corrects water velocity measurement errors associated with ultrasonic Doppler flow 
monitoring. The error identification is semi-automated against test criteria. The error correction procedure defines depth-velocity relationships from valid 'training data', then uses these to automatically replace erroneous velocities given valid depth data. The methodology is practical, consistent and updateable. It is a significant advance over previous UDFM data correction methods. The method maximises the value of existing data, requiring no additional data collection. Used in 'routine' mode for ongoing data collection campaigns, it can highlight changes in instrument or site behaviour at the earliest opportunity.

(2) The methodology has been applied to two example sites: a stream culvert and a surface water drainage pipe. Gaps in the discharge series, caused by 'default zero velocity' errors, have been filled and 'default repeat velocity' errors have been replaced. This provides a qualitative improvement over direct use of the raw data.

(3) The methodology improves the applicability of UDFM to smaller discharges, surface water ('clean') flows, backed up conditions and sub-optimal sites.

(4) Future work could include refinement of the depth-velocity relationships, e.g. accounting for flow hysteresis and transitions between sub- and super- critical flow.

\section{Acknowledgements}

The work presented in this paper has been developed under Water Cycle Management for New Developments (WaND) and the project support from EPSRC and industrial collaborators acknowledged. NERC provided additional support. We would like to thank: Tony Porter, Ian Gowing and Amanda Lloyd for fieldwork assistance; Oxfordshire County Council, Bryant Homes and Thames Water for monitoring permissions; the anonymous reviewers for helpful comments. 


\section{References}

ADS (2007) Flow Monitors and Communications [online]. ADS. http://www.adsenv.com/default.aspx?id=21 [accessed 21 June 2007]

BSI (1992) ISO/TR 9824-1: Measurement of liquid flow in open channels. Part 11.

Free surface flow in closed conduits. Part 11A. Methods of measurement. BSI.

CEH (2006) HYDATA - Hydrological Database and Analysis software [online].

CEH. http://www.ceh.ac.uk/products/software/CEHSoftware-HYDATA.htm [accessed 30 October 2006]

Enfinger, K. L. \& Kimbrough, H. R. (2004) Scattergraph Principles and Practice: A Comparison of Various Applications of the Manning Equation. In Galleher Jr., J. J. \& Stift, M. T. (Eds.) Pipelines 2004: Pipeline Engineering and Construction: What's on the Horizon? pp. 50. American Society of Civil Engineers, San Diego, California, USA.

Enfinger, K. L. \& Schutzbach, J. S. (2005) Scattergraph Principles and Practice: Camp's Varying Roughness Coefficient Applied to Regressive Methods. In Vipulanandan, C. \& Ortega, R. (Eds.) Pipelines 2005: Optimizing Pipeline Design, Operations, and Maintenance in Today's Economy. pp. 72-83. American Society of Civil Engineers, Houston, TX, USA.

Enfinger, K. L. \& Stevens, P. L. (2006) Scattergraph Principles and Practice: Tools and Techniques to Evaluate Sewer Capacity. In Atalah, A. \& Tremblay, A. (Eds.) Pipelines 2006: Service to the Owner. pp. 16. American Society of Civil Engineers, Chicago, Illinois, USA.

Herschy, R. W. (1995) Streamflow measurement, Second ed. E\&FN Spon, London. Hydro-Logic (2006) HYDROLOG4 [online]. Hydro-Logic. http://www.hydrologic.co.uk/content/view/14/50/ [accessed 30 October 2006]

Packman, J. C. \& Hewitt, E. J. (2000) Urban runoff control in the developing world. Appendix A. Data processing procedures. Department for International Development, UK.

Petersen-Overleir, A. (2006) Modelling stage-discharge relationships affected by hysteresis using the Jones formula and nonlinear regression. Hydrological Sciences Journal-Journal Des Sciences Hydrologiques, 51 (3), 365-388.

Unidata (2000) STARFLOW Ultrasonic Doppler Instrument User's Manual, Unidata, Australia.

Vermeyen, T. B. (2000) A Laboratory Evaluation of Unidata's Starflow Doppler Flowmeter and MGD Technologies Acoustic Doppler Flow Meter (ADFM) [online]. USBR.

http://www.usbr.gov/pmts/hydraulics_lab/tvermeyen/starflow/index.html [accessed 3 January 2007]

WaND (2007) Water Cycle Management for New Developments [online]. WaND. http://www.wand.uk.net/index.php [accessed 23 January 2007]

Watt, I. A. \& Jefferies, C. (1996) Portable sewage flow sensors - Their calibration and accuracy. Water Science and Technology, 33 (1), 127-137.

WRc (1987) A Guide to Short Term Flow Surveys of Sewer Systems. Water Research Centre, Swindon. 


\section{Word count}

4060 (main text and figure captions)

\section{Figure Captions}

Fig. 1. Site A: Scatter plot of raw data showing typical errors.

Fig. 2. Site B: Scatter plot of raw data showing typical errors, with inset detail for low depths.

Fig. 3. Site A: Scatter plot of cleaned training data.

Fig. 4. Site B: Scatter plot of cleaned training data, with inset detail for low depths.

Fig. 5. Site A: Plot of cleaned training data showing the regression relationship (solid line), deviations and the sub-/super- critical flow boundary (dashed line).

Fig. 6. Site A: Smoothed velocity time-series (line) for a large event, compared to depth-velocity data (points).

Fig. 7. Site B: Logarithmic plot of cleaned training data showing three zones (delineated by dash-dot thresholds lines), the predominant depth-velocity relationship in each zone (solid trend line(s)) and the sub-/super- critical flow boundary (dashed line). 
Fig. 8. Site B: Histogram of low velocities from cleaned training data.

Fig. 9. Flowchart showing data correction process used in INFILL_SF for Site B.

Fig. 10. Data processing program use in training and routine modes (dotted line shows use of previous output).

Fig. 11. Site A: Time series of depth and velocity (raw \& corrected).

Fig. 12. Site B: Time series of depth and velocity (raw \& corrected) under normal flow conditions.

Fig. 13. Site B: Time series of depth and velocity (raw \& corrected) under backed up flow conditions.

Fig. 14. Site A: Time series of discharge (raw \& corrected).

Fig. 15. Site B: Time series of discharge (raw \& corrected).

Fig. 16. Site B: Time series of discharge (raw \& corrected). 